\title{
Comparison of distribution characteristics of metastatic bone lesions between breast and prostate carcinomas
}

\author{
CHANG-YIN WANG ${ }^{1}$, GUANG-YAO WU ${ }^{2}$, MEI-JUAN SHEN ${ }^{1}$, KUN-WEI CUI ${ }^{1}$ and YING SHEN ${ }^{1}$ \\ Departments of ${ }^{1}$ Nuclear Medicine and ${ }^{2}$ Radiology, Zhongnan Hospital of Wuhan University, Wuhan 430071, Hubei, P.R. China
}

Received July 23, 2012; Accepted October 17, 2012

DOI: $10.3892 / 01.2012 .1005$

\begin{abstract}
The purpose of the present study was to investigate the distribution characteristics of bone metastases in breast and prostate carcinomas. Bone scintigraphies were performed in 504 cancer patients. We studied the correlation between the distribution and total number of metastatic bone lesions, and compared the distribution of metastatic bone lesions between breast and prostate carcinomas. In the early stage, the distribution in the thoracic vertebrae, lumbar vertebrae and pelvis of the metastatic lesions of the prostate carcinoma $(81.0 \%, 47 / 58)$ was significantly higher than that of the breast carcinoma $\left(41.7 \%, 63 / 151 ; \chi^{2}=27.6\right.$, $\mathrm{P}=0.000)$. The distribution of the lesions in the thoracic skeleton in the cases of the breast carcinoma $(65.6 \%, 99 / 151)$ was significantly higher than that of the prostate carcinoma $(27.6 \%$, $16 / 58 ; \chi^{2}=24.8, \mathrm{P}=0.000$ ); however, the distributions in the advanced cases were not markedly different. The differences in the proportions of the metastatic lesions in the lumbar vertebrae $\left(\chi^{2}=56.1, \mathrm{P}=0.000\right)$ and ribs $\left(\chi^{2}=39.1, \mathrm{P}=0.000\right)$ in the cases of the prostate carcinoma, and in the sternum $\left(\chi^{2}=31.2, \mathrm{P}=0.000\right)$, skull $\left(\chi^{2}=26.5, \mathrm{P}=0.000\right)$ and femur $\left(\chi^{2}=13.6, \mathrm{P}=0.001\right)$ in the cases of the breast carcinoma were significant. Between the breast and prostate carcinomas, the differences in the proportions of the metastatic lesions of certain bones were also significant. In cases with few bone metastases, the proportion of sternum metastases of patients with breast carcinoma (17.9\%) was significantly higher than that of patients with prostate carcinoma $\left(1.7 \% ; \chi^{2}=12.7\right.$, $\mathrm{P}=0.000)$; the proportion of metastases in the lumbar vertebrae of prostate carcinoma $(39.7 \%)$ was significantly higher than that of breast carcinoma $\left(13.9 \% ; \chi^{2}=15.4, \mathrm{P}=0.000\right)$; the proportion of rib metastases of breast carcinoma $(27.2 \%)$ was significantly higher than that of prostate carcinoma $\left(8.6 \% ; \chi^{2}=9.6, \mathrm{P}=0.002\right)$. In cases with extensive bone metastases, the proportions of metastatic lesions in the sternum and lumbar vertebrae in breast and prostate carcinomas were not significantly different $(\mathrm{P}>0.05)$. In conclusion, the distribution of bone metastases is correlated with
\end{abstract}

Correspondence to: Dr Chang-Yin Wang, Department of Nuclear Medicine, Zhongnan Hospital of Wuhan University, 169 DongHu Road, ShuiGuoHu, Wuhan 430071, Hubei, P.R. China E-mail: changyinwang@rocketmail.com

Key words: breast neoplasms, prostatic neoplasms, neoplasm metastasis, bone scan the total number of metastatic bone lesions in breast and prostate carcinoma patients, and has different characteristics in different lesions.

\section{Introduction}

Prostate and breast carcinomas are clinically common malignancies and often progress to bone metastasis (1-4). Bone scintigraphy is a sensitive and efficient method for the detection of bone metastases of malignant tumors, although it is considered to be a nonspecific method. Bone scintigraphy has value in determining the stage (1), selecting therapeutic schedule, evaluating prognosis (2) and performing follow-up (3) for tumor patients. A number of studies concerning bone scintigraphy of these tumors have been reported in the literature, but a comparative study of the distribution of metastatic bone lesions in prostate and breast carcinomas has not been performed. In several previous studies (4-6), Tofe et al (5) compared the distribution of metastatic skeletal involvements of different tumors by patient-based analyses. However, the total incidences of bone metastasis in the tumor patients have significant differences (breast carcinoma, 28 vs. $67 \%$; prostate carcinoma, 35 vs. $62 \%$ ) as demonstrated in previous studies $(5,6)$. If the total incidence of bone metastasis of a certain tumor is very high, the metastasis incidence of each category of bones (such as the ribs and vertebrae) of the tumor is correspondingly high. Accordingly, when the metastases of bones within the same bone category are compared in different tumors, the total incidences of bone metastasis may influence the results. Furthermore, the incidence of bone metastasis cannot represent the constituent ratio and distribution of certain metastatic bone lesions in the entire metastatic bone lesion. The incidence of bone metastasis was determined by patient-based analysis and was equal to the ratio of the number of patients who had bone metastasis to the number of total patients. Hence, it is not reasonable to evaluate the distribution of the metastatic bone lesions by the incidence. The results of other studies have shown that predilection sites and total distribution characteristics of bone metastases were similar in prostate $(7,8)$ and breast $(5,9,10)$ carcinomas; the ribs, thoracic vertebrae, lumbar vertebrae and ilium were reported to be the main predilection sites for the two diseases. However, the results of the study by Conti et al (2) indicated that the femur of prostate carcinoma patients was the most frequent bone metastatic site, which is different from the result of Huang et al (7). It is thus clear that there are controversies surrounding the distribution of bone metastases. 
We studied the distribution of bone metastases of breast and prostate carcinomas using the lesion-based analysis method. We classified the patients with metastatic bone diseases according to the total number of metastatic bone lesions, and studied the changes in distribution of metastatic bone lesions in cases with different numbers of lesions. We also compared the distributions between the two types of carcinoma. We found that the distribution of metastatic bone lesions in breast and prostate carcinomas was correlated with the lesion number and also had different characteristics.

\section{Patients and methods}

Patients. In this study, 504 patients in whom bone scintigraphies were performed were enrolled, including 360 patients with breast carcinoma (4 males; 356 females; ages, 22-82 years; median age, 50.0 years) and 144 male patients with prostate carcinoma (ages, 38-92 years; median age, 72.5 years). All patients received a definitive diagnosis using a pathological method. Only one bone scintigraphy was registered for study. If necessary, the other bone scintigraphies, performed before and after the registered bone scintigraphy, were regarded as references for diagnosis and were not registered. The clinical study was not limited to a specific stage of disease. This study was approved by the local Ethics Committees of Zhongnan Hospital of Wuhan University, Wuhan, China, and informed consent was obtained from all participants.

Imaging. SPECT with low-energy and high-resolution collimator (Model \#, E.CAM; Siemens Company, Hoffman Estates, IL, USA), ${ }^{99} \mathrm{Mo}-{ }^{-99 m} \mathrm{Tc}$ generators and the methylene diphosphonate (Beijing Institute of Atomic Energy in China, Beijing, China) were used in this study. All patients were instructed to drink $\sim 1,000 \mathrm{ml}$ water within $2 \mathrm{~h}$ of the intravenous injection of $740 \mathrm{MBq}$ of ${ }^{99 \mathrm{~m}} \mathrm{Tc}-\mathrm{methylene}$ diphosphonate and to urinate prior to bone scintigraphy. All planar bone scans, including anterior and posterior projections, were performed $\sim 3 \mathrm{~h}$ after the injection of imaging reagents, according to the following conditions: supine posture of patients; matrix, 256 x 1024; zoom, 1.0; movement velocity of examination bed, $15-25 \mathrm{~cm} / \mathrm{min}$. If necessary, additional local planar and SPECT bone imaging were performed.

Diagnostic criteria of bone metastasis. The interpretations of the ${ }^{99 \mathrm{~m}} \mathrm{Tc}-\mathrm{MDP}$ planar bone scan and SPECT bone imaging were made as a consensus reading of two nuclear medicine physicians, and that of the X-ray, CT and MRI as a consensus reading of a nuclear medicine physician and a radiological physician. According to the diagnostic method described by Even-Sapir et al (11), the results of the bone scans were interpreted as metastatic bone lesions under these circumstances: i) Vertebral lesions of increased uptake involved the posterior aspect of the vertebral body and pedicle, or involved the vertebrae extensively $(12,13)$; ii) rib lesions of increased uptake exhibited the strip form (14); iii) the lesions of increased uptake exhibited the lamellar form with or without corroding multiple adjacent bones (14); iv) the lesions of decreased uptake were confirmed to not be bone cysts and ischemic bone necrosis, or accompanied progressive osteodynia symptoms; v) the lesions of markedly increased uptake were confirmed to not be benign bone diseases, or exhibited multiple sites and scrambled arrangement; vi) the bone lesions, of increased or decreased uptake, were confirmed to be bone metastases by X-ray, CT or MRI; vii) by comparing the result of bone scans that were performed at different times, the new bone lesions could not be interpreted as benign diseases, or the lesions presented 'flare phenomenon' (15). The results of bone scans were considered negative under the following circumstances: i) no abnormal radiotracer uptake was detected; ii) there was indeterminate decreased radioactivity in local bones; iii) the bone lesions of increased or decreased radiotracer uptake were characterized as benign disease.

Relevant data were collected by direct interviews or from medical files on the trauma and surgical information in order to exclude the possibility that the benign lesions were caused by the surgery or trauma, and if necessary, the diagnosis was decided by follow-up after more than six months. For patients with fewer lesions or with vertebral lesions, especially those with only lumbar lesions, the results of the bone X-ray, CT, MRI, local planar bone imaging, SPECT bone imaging and previous bone scintigraphies were comprehensively analyzed. The aim was to exclude the common benign diseases of bones and joints, including hyperosteogeny, osteophytes, bone-bridge, degenerative osteoarthropathy and inflammatory disease, as much as possible.

Grouping. To study the correlation between the distribution and the number of metastatic bone lesions and to compare the distribution of the metastatic bone lesions between breast and prostate carcinomas, we divided patients with bone metastasis into three groups according to the total number of metastatic bone lesions: few bone metastases, with the total number being 1-3; moderate bone metastases, with the total number being 4-10; extensive bone metastases, with the total number being $>10$.

Statistical analyses. Data were analyzed using the SPSS statistical software (version 13.0; SPSS, Inc., Chicago, IL, USA). The Chi-square test was performed to compare the difference between the proportions. $\mathrm{P}<0.05$ was considered to indicate a statistically significant result.

\section{Results}

Incidence of bone metastasis. Of a total of 504 patients with malignant tumors, 252 developed bone metastases and a total of 3,324 metastatic bone lesions were detected. The incidence of bone metastasis of prostate carcinoma $(70.8 \%$; 102/144) was higher than that of breast carcinoma $\left(41.7 \% ; 150 / 360 ; \chi^{2}=35.8\right.$, $\mathrm{P}=0.000)$.

Comparison of bone metastasis distribution between breast and prostate carcinomas. The proportions of metastatic bone lesions of breast and prostate carcinomas are listed in Tables I-IV. In cases with few bone metastases (Table I), the proportions of metastatic lesions of the lumbar vertebrae, ischium, pubis and femur in prostate carcinoma patients were higher than those in breast carcinoma patients; the proportions of metastatic lesions of the ribs, sternum and skull in breast carcinoma patients were higher than those in prostate carci- 
Table I. Comparison of bone metastases $(n=209)$ in cases with few bone metastases in breast and prostate carcinomas.

\begin{tabular}{|c|c|c|c|c|c|c|}
\hline \multirow[b]{2}{*}{ Skeleton } & \multicolumn{2}{|c|}{$\begin{array}{c}\text { Prostate } \\
\text { carcinoma }(n=58)\end{array}$} & \multicolumn{2}{|c|}{$\begin{array}{c}\text { Breast } \\
\text { carcinoma }(n=151)\end{array}$} & \multirow[b]{2}{*}{$\chi^{2}$} & \multirow[b]{2}{*}{ P-value } \\
\hline & $\mathrm{n}$ & $\%$ & $\mathrm{n}$ & $\%$ & & \\
\hline Cervical vertebrae & 2 & 3.45 & 3 & 1.99 & 0.357 & 0.550 \\
\hline Thoracic vertebrae & 10 & 17.24 & 24 & 15.89 & 0.055 & 0.814 \\
\hline Lumbar vertebrae & 23 & 39.66 & 21 & 13.91 & 15.431 & 0.000 \\
\hline Sacrococcyx & 2 & 3.45 & 6 & 3.97 & 0.032 & 0.858 \\
\hline Ilium & 6 & 10.34 & 11 & 7.29 & 0.502 & 0.478 \\
\hline Ischium & 2 & 3.45 & 0 & 0.00 & 5.178 & 0.023 \\
\hline Pubis & 4 & 6.90 & 1 & 0.66 & 6.070 & 0.014 \\
\hline Ribs & 5 & 8.62 & 41 & 27.15 & 9.632 & 0.002 \\
\hline Sternum & 1 & 1.72 & 27 & 17.88 & 12.719 & 0.000 \\
\hline Skull & 0 & 0.00 & 6 & 3.97 & 3.968 & 0.046 \\
\hline Scapula & 0 & 0.00 & 4 & 2.65 & 2.630 & 0.105 \\
\hline Collar bone & 0 & 0.00 & 3 & 1.99 & 1.967 & 0.161 \\
\hline Humerus & 0 & 0.00 & 3 & 1.99 & 1.967 & 0.161 \\
\hline Femur & 3 & 5.17 & 1 & 0.66 & 3.930 & 0.047 \\
\hline Radioulnar and tibiofibular & 0 & 0.00 & 0 & 0.00 & - & - \\
\hline Bones of hands and feet & 0 & 0.00 & 0 & 0.00 & - & - \\
\hline
\end{tabular}

$\mathrm{n}$, the lesion number of bone metastases. Chi-square test of likelihood ratio was performed to compare the proportions of metastatic bone lesions in breast and prostate carcinomas.

Table II. Comparison of bone metastasis distribution between breast and prostate carcinomas.

\begin{tabular}{|c|c|c|c|c|c|c|c|c|c|c|c|c|}
\hline \multirow[b]{3}{*}{ Metastatic position } & \multicolumn{6}{|c|}{ Few bone metastases } & \multicolumn{6}{|c|}{ Extensive bone metastases } \\
\hline & \multicolumn{2}{|c|}{ Bca $(n=151)$} & \multicolumn{2}{|c|}{$\operatorname{Pca}(n=58)$} & \multirow[b]{2}{*}{$\chi^{2}$} & \multirow[b]{2}{*}{ P-value } & \multicolumn{2}{|c|}{ Bca $(n=989)$} & \multicolumn{2}{|c|}{ Pca $(n=1781)$} & \multirow[b]{2}{*}{$\chi^{2}$} & \multirow[b]{2}{*}{$\mathrm{P}$-value } \\
\hline & $\mathrm{n}$ & $\%$ & $\mathrm{n}$ & $\%$ & & & $\mathrm{n}$ & $\%$ & $\mathrm{n}$ & $\%$ & & \\
\hline Vertebrae and pelvis & 63 & 41.7 & 47 & 81.0 & 27.6 & 0.000 & 405 & 41.0 & 866 & 48.6 & 15.1 & 0.000 \\
\hline Thoracic skeleton & 99 & 65.6 & 16 & 27.6 & 24.8 & 0.000 & 498 & 50.4 & 919 & 51.6 & 0.4 & 0.530 \\
\hline
\end{tabular}

$\mathrm{n}$, the lesion number of bone metastases; Bca, breast carcinoma; Pca, prostate carcinoma. Vertebrae and pelvis includes the thoracic vertebrae, lumbar vertebrae, sacrococcyx, ilium, ischium and pubis. Thoracic skeleton includes the sternum, collar bone, ribs, scapula and thoracic vertebrae. Chi-square test of likelihood ratio was performed to compare the proportions of metastatic bone lesions in breast and prostate carcinomas.

noma patients. In cases of prostate carcinoma, $81.0 \%(47 / 58)$ of the metastatic bone lesions were located in the thoracic vertebrae, lumbar vertebrae and pelvis, whereas in breast carcinoma, $65.6 \%(99 / 151)$ of lesions were located in the thoracic skeleton (Table II). In cases with moderate bone metastases (Table III), the proportions of metastatic lesions of the ribs and sternum in breast carcinoma patients were higher than those in prostate carcinoma patients. In cases with extensive bone metastases (Table IV), the proportions of metastatic lesions of the ilium, ischium, pubis and ribs in prostate carcinoma patients were higher than those in breast carcinoma patients; the proportion of metastatic lesions of the skull in breast carcinoma patients was higher than that in prostate carcinoma patients; however, the proportions of metastatic lesions of the lumbar vertebrae, sternum and femur were not significantly different between breast and prostate carcinomas.
Change of predilection sites in cases with different numbers of lesions. The results showed that the ribs, thoracic vertebrae, lumbar vertebrae and ilium were predilection sites of bone metastases, but on the basis of the proportions, the sequences of these predilection sites were different in cases with different numbers of lesions and in different tumors. For prostate carcinoma, in decreasing order, the predilection sites of bone metastasis were the lumbar vertebrae, thoracic vertebrae, ilium and ribs in cases with few bone metastases (Table I); the thoracic vertebrae, lumbar vertebrae, ilium and ribs in cases with moderate bone metastases (Table III); and the ribs, ilium, thoracic vertebrae and lumbar vertebrae in cases with extensive bone metastases (Table IV). For breast carcinoma, in decreasing order, the predilection sites of bone metastasis were the ribs, sternum, thoracic vertebrae, lumbar vertebrae and ilium in cases with few bone metastases (Table II). The ribs, thoracic vertebrae, lumbar vertebrae, ilium 
Table III. Comparison of bone metastases $(n=345)$ in cases with moderate bone metastases in breast and prostate carcinomas.

\begin{tabular}{|c|c|c|c|c|c|c|}
\hline \multirow[b]{2}{*}{ Skeleton } & \multicolumn{2}{|c|}{$\begin{array}{c}\text { Prostate } \\
\text { carcinoma }(n=161)\end{array}$} & \multicolumn{2}{|c|}{$\begin{array}{c}\text { Breast } \\
\text { carcinoma }(n=184)\end{array}$} & \multirow[b]{2}{*}{$\chi^{2}$} & \multirow[b]{2}{*}{ P-value } \\
\hline & $\mathrm{n}$ & $\%$ & $\mathrm{n}$ & $\%$ & & \\
\hline Cervical vertebrae & 5 & 3.11 & 0 & 0.00 & 7.705 & 0.006 \\
\hline Thoracic vertebrae & 39 & 24.22 & 34 & 18.48 & 1.696 & 0.193 \\
\hline Lumbar vertebrae & 22 & 13.66 & 24 & 13.04 & 0.029 & 0.866 \\
\hline Sacrococcyx & 14 & 8.70 & 8 & 4.35 & 2.730 & 0.098 \\
\hline Ilium & 22 & 13.66 & 23 & 12.50 & 0.103 & 0.749 \\
\hline Ischium & 11 & 6.83 & 10 & 5.43 & 0.293 & 0.589 \\
\hline Pubis & 7 & 4.35 & 4 & 2.17 & 1.320 & 0.251 \\
\hline Ribs & 22 & 13.66 & 53 & 28.80 & 11.908 & 0.001 \\
\hline Sternum & 1 & 0.62 & 14 & 7.61 & 12.215 & 0.000 \\
\hline Skull & 5 & 3.11 & 2 & 1.09 & 1.796 & 0.180 \\
\hline Scapula & 0 & 0.00 & 4 & 2.17 & 5.070 & 0.024 \\
\hline Collar bone & 1 & 0.62 & 2 & 1.09 & 0.222 & 0.638 \\
\hline Humerus & 5 & 3.11 & 3 & 1.63 & 0.827 & 0.363 \\
\hline Femur & 7 & 4.35 & 3 & 1.63 & 2.290 & 0.130 \\
\hline Radioulnar and tibiofibular & 0 & 0.00 & 0 & 0.00 & - & - \\
\hline Bones of hands and feet & 0 & 0.00 & 0 & 0.00 & - & - \\
\hline
\end{tabular}

$\mathrm{n}$, the lesion number of bone metastases. Chi-square test of likelihood ratio was performed to compare the proportions of metastatic bone lesions in breast and prostate carcinomas.

Table IV. Comparison of bone metastases $(n=2770)$ in cases with extensive bone metastases in breast and prostate carcinomas.

\begin{tabular}{|c|c|c|c|c|c|c|}
\hline \multirow[b]{2}{*}{ Skeleton } & \multicolumn{2}{|c|}{$\begin{array}{c}\text { Prostate } \\
\text { carcinoma }(n=1781)\end{array}$} & \multicolumn{2}{|c|}{$\begin{array}{c}\text { Breast } \\
\text { carcinoma }(n=989)\end{array}$} & \multirow[b]{2}{*}{$\chi^{2}$} & \multirow[b]{2}{*}{ P-value } \\
\hline & $\mathrm{n}$ & $\%$ & $\mathrm{n}$ & $\%$ & & \\
\hline Cervical vertebrae & 53 & 2.98 & 37 & 3.74 & 1.162 & 0.281 \\
\hline Thoracic vertebrae & 247 & 13.87 & 135 & 13.65 & 0.026 & 0.873 \\
\hline Lumbar vertebrae & 115 & 6.46 & 71 & 7.18 & 0.524 & 0.469 \\
\hline Sacrococcyx & 80 & 4.49 & 37 & 3.74 & 0.902 & 0.342 \\
\hline Ilium & 248 & 13.92 & 97 & 9.81 & 10.206 & 0.001 \\
\hline Ischium & 96 & 5.39 & 36 & 3.64 & 4.467 & 0.035 \\
\hline Pubis & 80 & 4.49 & 29 & 2.93 & 4.277 & 0.039 \\
\hline Ribs & 551 & 30.94 & 269 & 27.20 & 4.297 & 0.038 \\
\hline Sternum & 56 & 3.14 & 43 & 4.35 & 2.604 & 0.107 \\
\hline Skull & 60 & 3.37 & 97 & 9.81 & 46.771 & 0.000 \\
\hline Scapula & 44 & 2.47 & 37 & 3.74 & 3.502 & 0.061 \\
\hline Collar bone & 21 & 1.18 & 14 & 1.42 & 0.280 & 0.596 \\
\hline Humerus & 36 & 2.02 & 33 & 3.34 & 4.363 & 0.037 \\
\hline Femur & 90 & 5.05 & 52 & 5.26 & 0.054 & 0.815 \\
\hline Radioulnar and tibiofibular & 4 & 0.22 & 2 & 0.20 & 0.015 & 0.903 \\
\hline Bones of hands and feet & 0 & 0.00 & 0 & 0.00 & - & - \\
\hline
\end{tabular}

$\mathrm{n}$, the lesion number of bone metastases. Chi-square test of likelihood ratio was performed to compare the proportions of metastatic bone lesions in breast and prostate carcinomas.

and sternum in cases with moderate bone metastases (Table III); and the ribs, thoracic vertebrae, ilium, skull and lumbar vertebrae in cases with extensive bone metastases (Table IV).
Proportion of non-predilection sites in different lesions. The results in Tables I, III and IV show that the cervical vertebrae, scapula, collar bone and humerus did not have a 


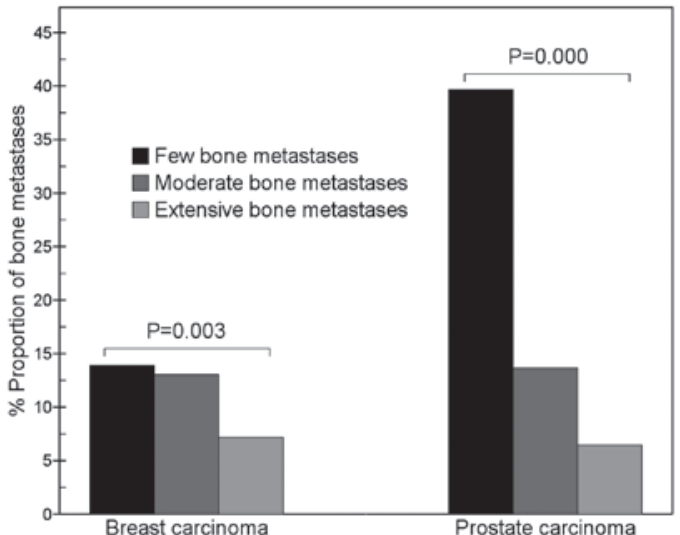

Figure 1. Comparison of lumbar vertebrae metastases between breast and prostate carcinomas. The results show that with an increase in the number of metastatic bone lesions, the proportion of metastatic lumbar lesions of breast carcinoma gradually decreased $\left(\chi^{2}=11.3, \mathrm{P}=0.003\right)$, but that in prostate carcinoma markedly decreased $\left(\chi^{2}=56.1, \mathrm{P}=0.000\right)$. In the few bone metastases group, the proportion of metastatic lumbar lesions of prostate carcinoma was significantly higher than that of breast carcinoma ( 39.7 vs. $13.9 \%, \chi^{2}=15.4, \mathrm{P}=0.000$ ).

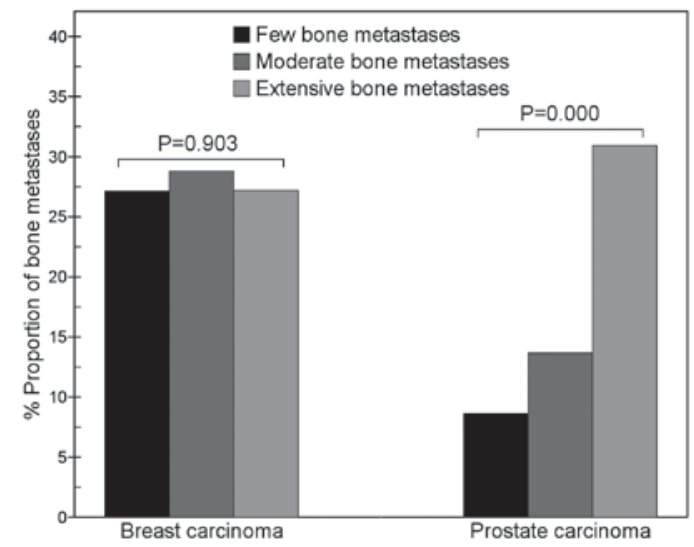

Figure 2. Comparison of rib metastases between breast and prostate carcinomas. The results show that with an increase in the number of metastatic bone lesions, the proportion of metastatic rib lesions of prostate carcinoma gradually increased $\left(\chi^{2}=39.1, \mathrm{P}=0.000\right)$, but that of breast carcinoma did not change $\left(\chi^{2}=0.2, \mathrm{P}=0.903\right)$. In the few bone metastases group, the proportion of metastatic rib lesions of prostate carcinoma was markedly lower than that of breast carcinoma ( 8.6 vs. $\left.27.2 \%, \chi^{2}=9.6, \mathrm{P}=0.002\right)$, but in the extensive bone metastases group, it was similar to that of breast carcinoma (30.9 vs. $\left.27.2 \%, \chi^{2}=4.3, \mathrm{P}=0.038\right)$.

higher proportion of bone metastasis in breast and prostate carcinomas compared to the predilection sites in the ribs, thoracic vertebrae, lumbar vertebrae and ilium. For prostate carcinoma, no metastatic bone lesion was detected in these bones, with the exception of the cervical vertebrae in the few bone metastases group (Table I). The metastatic lesion proportions of the radioulnar, tibiofibular and bones of the hands and feet were lowest in breast and prostate carcinomas, and no metastatic lesion was detected in these bones in either the few or moderate bone metastases groups, whereas only a small number of metastatic bone lesions was detected in the extensive bone metastases group. Therefore, the radioulnar, tibiofibular and bones of the hands and feet were non-predilection site of bone metastasis.

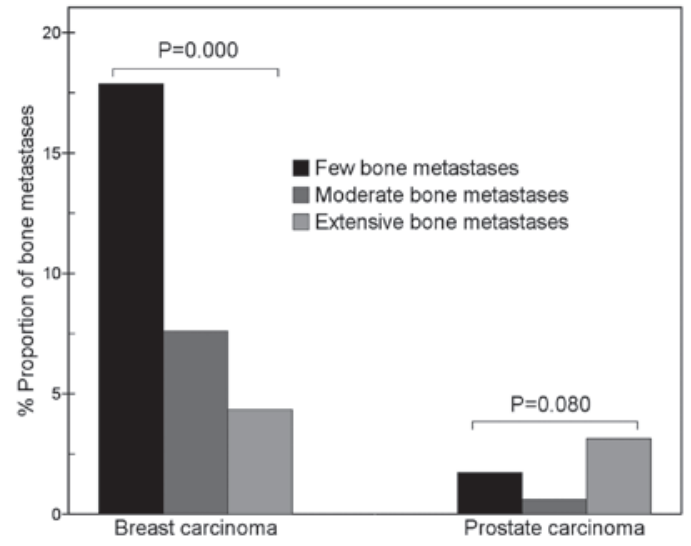

Figure 3. Comparison of sternum metastases between breast and prostate carcinomas. The results show that with an increase in the number of metastatic bone lesions, the proportion of metastatic sternum lesions of breast carcinoma markedly decreased $\left(\chi^{2}=31.2, \mathrm{P}=0.000\right)$, but that of prostate carcinoma did not change $\left(\chi^{2}=5.0, \mathrm{P}=0.080\right)$. In the few bone metastases group, the proportion of metastatic sternum lesions of breast carcinoma was significantly higher than that of prostate carcinoma $\left(17.9\right.$ vs. $\left.1.7 \%, \chi^{2}=12.7, \mathrm{P}=0.000\right)$.

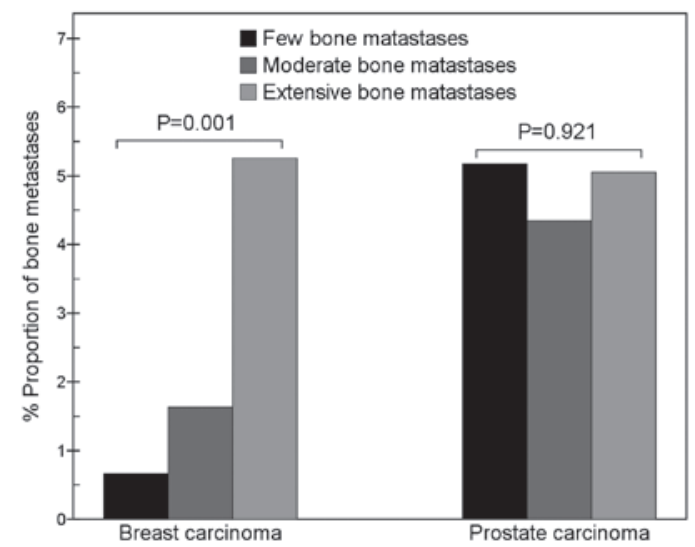

Figure 4. Comparison of femur metastases between breast and prostate carcinomas. The results show that with an increase in the number of metastatic bone lesions, the proportion of metastatic femur lesions of breast carcinoma gradually increased $\left(\chi^{2}=13.6, \mathrm{P}=0.001\right)$, but that of prostate carcinoma did not change $\left(\chi^{2}=0.2, \mathrm{P}=0.921\right)$. In the few bone metastases group, the proportion of metastatic femur lesions of breast carcinoma was significantly lower than that of prostate carcinoma $\left(\chi^{2}=3.9, \mathrm{P}=0.047\right)$.

Comparison of bone metastasis distribution in cases with different numbers of lesions. For prostate carcinoma, between the three groups with different numbers of lesions, the differences in the proportion of lesions in the thoracic vertebrae $\left(\chi^{2}=11.3, \mathrm{P}=0.003\right)$, lumbar vertebrae $\left(\chi^{2}=56.1, \mathrm{P}=0.000\right)$, ribs $\left(\chi^{2}=39.1, \mathrm{P}=0.000\right)$ and scapula $\left(\chi^{2}=10.3, \mathrm{P}=0.006\right)$ were significant. The proportion of metastatic lumbar vertebrae lesions in the few bone metastases group $(39.7 \% ; 23 / 58)$ was significantly higher than that in the moderate bone metastases group $(13.7 \%$; $\left.22 / 161 ; \chi^{2}=16.1, \mathrm{P}=0.000\right)$; and that in the moderate bone metastases group (13.7\%) was significantly higher than that in the extensive bone metastases group $\left(6.5 \% ; 115 / 1781 ; \chi^{2}=9.6\right.$, $\mathrm{P}=0.002$; Fig. 1). The proportion of metastatic rib lesions in the extensive bone metastases group $(30.9 \%$; 551/1781) was significantly higher than that in the moderate bone metastases group $\left(13.7 \% ; 22 / 161 ; \chi^{2}=24.2, \mathrm{P}=0.000\right)$ and that in the few bone metastases group $\left(8.6 \% ; 5 / 58 ; \chi^{2}=16.5, \mathrm{P}=0.000 ;\right.$ Fig. 2$)$. 
For breast carcinoma, metastases in the cervical vertebrae $\left(\chi^{2}=13.5, \mathrm{P}=0.001\right)$, lumbar vertebrae $\left(\chi^{2}=11.3, \mathrm{P}=0.003\right)$, ischium $\left(\chi^{2}=12.6, \mathrm{P}=0.002\right)$, sternum $\left(\chi^{2}=31.2, \mathrm{P}=0.000\right)$, skull $\left(\chi^{2}=26.5, \mathrm{P}=0.000\right)$ and femur $\left(\chi^{2}=13.6, \mathrm{P}=0.001\right)$ were present in significantly different proportions in cases with different numbers of bone metastases. The proportion of metastatic sternum lesions in the few bone metastases group (17.9\%; 27/151) was significantly higher than that in the moderate bone metastases group $\left(7.6 \% ; 14 / 184 ; \chi^{2}=8.2, \mathrm{P}=0.004\right)$ and that in the extensive bone metastases group $\left(4.3 \% ; 43 / 989 ; \chi^{2}=30.7\right.$, $\mathrm{P}=0.000$; Fig. 3). The proportion of metastatic femur lesions gradually increased with an increase in the total number of metastatic bone lesions (Fig. 4).

\section{Discussion}

Predilection and non-predilection sites of bone metastases. The most common human carcinomas (breast and prostate) have a great avidity for bone, frequently leading to painful and untreatable consequences. Patients with advanced breast and prostate carcinomas frequently develop bone metastases, and the risks are extremely high (16). There is a wide range in the reported incidence of bone metastasis. In the present study, the incidence of bone metastasis of prostate carcinoma $(70.8 \%)$ was higher than that of breast carcinoma (41.7\%). The study by Wilson and Calhoun (6) reported that the difference in bone metastasis incidence between breast and prostate carcinomas was not significant ( 28 vs. $35 \%, \chi^{2}=0.4, P=0.529$ ). In the study by Tofe et al (5), the incidences of bone metastasis of breast and prostate carcinomas were higher than that reported by Wilson and Calhoun and were similar in the two types of carcinoma (67 vs. $62 \%, \chi^{2}=1.3, \mathrm{P}=0.258$ ). Therefore, our result is different from the outcomes reported by Wilson and Calhoun (6) and by Tofe et al (5), which may be due to the different study populations.

Our results show that the ribs, thoracic vertebrae, lumbar vertebrae and ilium are the main predilection sites of breast and prostate carcinomas and that the middle-distal bones of the extremities are non-predilection sites, which are the common features of the distribution of metastatic bone lesions. The features are similar to the results reported in previous studies (5-10). Metastatic bone lesions are commonly distributed in these predilection sites and are rarely observed in the middle-distal bones of the extremities.

Correlation between distribution and number of metastatic bone lesions. In the present study, the proportion of metastatic lesions in certain bones was significantly different in cases with different numbers of metastatic bone lesions. With an increase in the number of metastatic bone lesions, the proportion of rib lesions in prostate carcinoma patients and that of femur lesions in breast carcinoma patients gradually increased; by contrast, those of lumbar vertebrae lesions in prostate carcinoma patients and sternum lesions in breast carcinoma patients gradually decreased. Accordingly, with regards to the proportion of metastatic bone lesions, the tendency of different bones to change varied. These outcomes demonstrate that the distribution of metastatic bone lesions is not always fixed, but is changed with an increase in the number of metastatic bone lesions. Therefore, the distribution of metastatic bone lesions is correlated with the number of bone lesions, and differs in cases with different numbers of metastatic bone lesions. This may be due to the non-random spreading of breast or prostate carcinoma cells, which do not spread to different bones proportionately, but prefer to metastasize to certain bones first and then to other bones via a different pathway.

Correlation between predilection site and number of metastatic bone lesions. As the distribution of metastatic bone lesions changes with the total number of lesions, the predilection sites of bone metastases may also be different in cases with different numbers of metastatic bone lesions. In the present study, the predilection sites of bone metastases or the sequence of the predilection sites were often different in cases with different numbers of lesions, which indicates that the predilection sites of bone metastases are correlated with the total number of metastatic bone lesions.

Patients with few bone metastases are considered to be in the early stage of bone metastases; patients with extensive bone metastases have a great number of metastases, so they are considered to have developed advanced stage bone metastases. Our results show that the lumbar vertebrae are the main predilection site of prostate carcinoma in the early stage, rather than the ribs. The hypothesis that tumor cells invade the lumbar vertebrae early in the disease and later metastasize to the ribs may be explained by spreading through the Batson venous plexus (17). Tumor cells usually spread to the lumbar vertebrae at an early stage via the Batson venous plexus, and then metastasize to the ribs and other bones; therefore, the ribs are the main predilection site of prostate carcinoma in advanced stages. However, the ribs of breast carcinoma patients are the most important predilection site in early and advanced stages, which is different from the distribution of rib lesions in prostate carcinoma patients. In the early stage, patients with breast carcinoma progress to rib metastasis more frequently than patients with prostate carcinoma. The reason for the difference may be different spreading mechanisms. Breast carcinoma cells may directly invade the neighboring ribs at an early stage and may also spread to the ribs via the aorta following metastasis to lung. However, the anatomical features of the prostate-Batson venous plexus path mean that prostate carcinoma cells are unable to reach the ribs at an early stage, but are able to at an advanced stage. These different mechanisms influence the early distribution of metastatic bone lesions, and so the early distribution of rib lesions is different in breast and prostate carcinoma. For breast carcinoma, after the ribs the sternum is the main predilection site of bone metastasis at early stages. Ohtake et al (18) suggested that breast carcinoma cells spread to the sternum via the parasternal lymph nodes, which is the reason that the sternum is invaded earlier by carcinoma cells than a number of other bones, except the ribs.

Different distributions of metastatic bone lesions in breast and prostate carcinoma. In the present study, most bone lesions of prostate carcinoma patients were distributed in the vertebrae and pelvis in the early stage, but those of breast carcinoma patients were located in the thoracic skeleton; therefore, the early distributions were different. Our results demonstrate that differences in the distribution of metastatic lesions of certain bones were present between breast and prostate carcinomas and also in cases with different numbers of lesions. In cases 
with few bone metastases, the proportion of metastatic lumbar lesions of prostate carcinoma (39.7\%) was approximately three times higher than that of breast carcinoma (13.9\%); by contrast, the proportion of metastatic rib lesions of breast carcinoma $(27.2 \%)$ was three to four times higher than that of prostate carcinoma (8.6\%). The results show that the distribution of early stage bone metastases in the lumbar vertebrae and ribs is markedly different in breast and prostate carcinomas. Furthermore, the distribution of early stage metastatic bone lesions in breast carcinoma is characterized by a markedly higher proportion of sternum metastases and a lower proportion of femur metastases than that of prostate carcinoma. These features are useful in the early and differential diagnosis of bone metastases in breast and prostate carcinomas. In cases with extensive bone metastases, a marked difference was observed in the proportion of breast carcinoma metastases to the skull, which was approximately three times higher than that in prostate carcinoma. The reason that the skull of breast carcinoma patients is more frequently invaded in the advanced stage of bone metastases may be that breast carcinoma cells spread to the skull by the lung-aorta route and also via the Batson venous plexus. The spreading to the skull of breast carcinoma cells may be easier than that of prostate carcinoma cells, via both the Batson venous plexus and lung-aorta paths. Therefore, the difference of predilection sites of bone metastases between breast and prostate carcinomas is most marked in cases with few bone metastases, but not in cases with extensive bone metastases.

Conclusion. The distributions of metastatic bone lesions in breast and prostate carcinomas not only have common features, but also have different characteristics. In the early stage, metastatic bone lesions of breast carcinoma are distributed mainly in the thoracic skeleton, but those of prostate carcinoma are mainly observed in the vertebrae and pelvis. The most noteworthy finding of the present study is that the distribution of bone metastases is correlated with the total number of metastatic bone lesions of patients. With an increase in the metastatic bone lesion number, the distribution of metastatic lesions of certain bones markedly changes, and the trend of the metastatic lesion distributions differs in certain bones or in two types of tumor. The bones which change most prominently are the ribs and lumbar vertebrae in prostate carcinoma and the sternum and femur in breast carcinoma. The proportion of metastatic bone lesions in the lumbar vertebrae in prostate carcinoma and the sternum in breast carcinoma markedly decreases with an increase in the total number of metastatic bone lesions; however, the proportions of lesions in the ribs in prostate carcinoma and the femur in breast carcinoma gradually increase with an increase in the number of lesions, which is different from many other bones. Our results indicate that the lumbar vertebrae in prostate carcinoma and the ribs and sternum in breast carcinoma are frequently invaded in the early stage of bone metastasis; however, the ribs in prostate carcinoma are seldom invaded in the early stage, but are invaded in the advanced stage. The difference of predilection sites between breast and prostate carcinomas is marked in cases with few bone metastases, but not in cases with extensive bone metastases. Understanding these different features of metastatic bone lesions in breast and prostate carcinomas is useful for an early and differential diagnosis of the diseases.

\section{Acknowledgements}

The authors thank Dr Xiang-yuan Zhang and Dr Ling-long Jiang for their technical assistance, and Dr Chenling Xiong from the Gene Diagnosis Center of Zhongnan Hospital for the assistance in the language editing.

\section{References}

1. Lin K, Szabo Z, Chin BB and Civelek AC: The value of a baseline bone scan in patients with newly diagnosed prostate cancer. Clin Nucl Med 24: 579-582, 1999.

2. Conti G, La Torre G, Cicalese V, et al: Prostate cancer metastases to bone: Observational study for the evaluation of clinical presentation, course and treatment patterns. Presentation of the METAURO protocol and of patient baseline features. Arch Ital Urol Androl 80: 59-64, 2008.

3. Maffioli L, Florimonte L, Pagani L, Butti I and Roca I: Current role of bone scan with phosphonates in the follow-up of breast cancer. Eur J Nucl Med Mol Imaging 31 (Suppl 1): S143-S148, 2004.

4. Osmond JD III, Pendergrass HP and Potsaid MS: Accuracy of ${ }^{99 \mathrm{~m} T c}$-diphosphonate bone scans and roentgenograms in the detection of prostate, breast and lung carcinoma metastases. Am J Roentgenol Radium Ther Nucl Med 125: 972-977, 1975.

5. Tofe AJ, Francis MD and Harvey WJ: Correlation of neoplasms with incidence and localization of skeletal metastases: An analysis of 1355 diphosphonate bone scans. J Nucl Med 16: 986-989, 1975.

6. Wilson MA and Calhoun FW: The distribution of skeletal metastases in breast and pulmonary cancer: concise communication. J Nucl Med 22: 594-597, 1981.

7. Huang CY, Hsu HC, Chang CH, Tseng KF and Fong YC: Prostate cancer with bone metastases: a clinical pofile. Mid Taiwan J Med 11: 82-89, 2006.

8. Picchio M, Spinapolice EG, Fallanca F, Crivellaro C, Giovacchini G, Gianolli L and Messa C: [11C] Choline PET/CT detection of bone metastases in patients with PSA progression after primary treatment for prostate cancer: comparison with bone scintigraphy. Eur J Nucl Med Mol Imaging 39: 13-26, 2012.

9. Jacobson AF, Stomper PC, Jochelson MS, Ascoli DM, Henderson IC and Kaplan WD: Association between number and sites of new bone scan abnormalities and presence of skeletal metastases in patients with breast cancer. J Nucl Med 31: 387-392, 1990.

10. Mohammadzadeh M, Alikhah $\mathrm{H}$ and Zareh AG: Comparison of bone scan with carbohydrate antigen 15-3 for evaluation of bone metastasis of breast cancer. Pak J Biol Sci 13: 175-179, 2010.

11. Even-Sapir E, Metser U, Mishani E, Lievshitz G, Lerman H and Leibovitch I: The detection of bone metastases in patients with high-risk prostate cancer: ${ }^{99 \mathrm{~m}} \mathrm{Tc}-\mathrm{MDP}$ Planar bone scintigraphy, single-and multi-field-of-view SPECT, ${ }^{18} \mathrm{~F}$-fluoride PET, and ${ }^{18}$ F-fluoride PET/CT. J Nucl Med 47: 287-297, 2006.

12. Gate GF: SPECT bone scanning of the spine. Semin Nucl Med 28: 78-94, 1998.

13. Even-Sapir E, Martin RH, Barnes DC, Pringle CR, Iles SE and Mitchell MJ: Role of SPECT in differentiating malignant from benign lesions in the lower thoracic and lumbar vertebrae. Radiology 187: 193-198, 1993.

14. Wang CY and Zhang XY: Evaluation of eikonic characteristic of skeletal metastasis of primary pulmonary carcinoma with ${ }^{99} \mathrm{Tc}^{\mathrm{m}}$ methylene diphosphonate whole-body bone scans. ChineseGerman J Clin Oncol 9: 435-440, 2010.

15. Cook GJ, Venkitaraman R, Sohaib AS, et al: The diagnostic utility of the flare phenomenon on bone scintigraphy in staging prostate cancer. Eur J Nucl Med Mol Imaging 38: 7-13, 2011.

16. Mundy GR: Metastasis to bone: causes, consequences and therapeutic opportunities. Nat Rev Cancer 2: 584-593, 2002.

17. Bubendorf L, Schöpfer A, Wagner U, et al: Metastatic patterns of prostate cancer: an autopsy study of 1,589 patients. Hum Pathol 31: $578-583,2000$.

18. Ohtake E, Murata $\mathrm{H}$ and Maruno $\mathrm{H}$ : Bone scintigraphy in patients with breast cancer: malignant involvement of the sternum. Radiat Med 12: 25-28, 1994. 